\title{
High-throughput genetic newborn screening for spinal muscular atrophy by rapid nucleic acid extraction from dried blood spots and 384-well qPCR
}

\author{
Ludwig Czibere $\mathbb{I}^{1}$ - Siegfried Burggraf $\mathbb{D}^{1} \cdot{\text { Tobias Fleige } \mathbb{D}^{1} \cdot \text { Birgit Glück }^{1} \cdot \text { Lisa Marie Keitel }}^{1} \cdot$ Olfert Landt $^{2}$. \\ Jürgen Durner ${ }^{1,3} \cdot$ Wulf Röschinger ${ }^{1} \cdot$ Katharina Hohenfellner $^{4} \cdot$ Brunhilde Wirth $^{5} \cdot$ Wolfgang Müller-Felber $^{6}$. \\ Katharina Vill ${ }^{6}$ Marc Becker ${ }^{1,3}$
}

Received: 21 March 2019 / Revised: 27 June 2019 / Accepted: 3 July 2019 / Published online: 30 July 2019

(c) The Author(s), under exclusive licence to European Society of Human Genetics 2019

\begin{abstract}
Establishing nucleic acid-based assays for genetic newborn screening (NBS) provides the possibility to screen for genetically encoded diseases like spinal muscular atrophy (SMA), best before the onset of symptoms. Such assays should be easily scalable to 384-well reactions that make the screening of up to 2000 samples per day possible. We developed a test procedure based on a cleanup protocol for dried blood spots and a quantitative (q)PCR to screen for a homozygous deletion of exon 7 of the survival of motor neuron 1 gene $(S M N 1)$ that is responsible for $>95 \%$ of SMA patients. Performance of this setup is evaluated in detail and tested on routine samples. Our cleanup method for nucleic acids from dried blood spots yields enough DNA for diverse subsequent qPCR applications. To date, we have applied this approach to test 213,279 samples within 18 months. Thirty patients were identified and confirmed, implying an incidence of 1:7109 for the homozygous deletion. Using our cleanup method, a rapid workflow could be established to prepare nucleic acids from dried blood spot cards. Targeting the exon 7 deletion, no invalid, false-positive, or false-negative results were reported to date. This allows timely identification of the disease and grants access to the recently introduced treatment options, in most cases before the onset of symptoms. Carriers are not identified, thus, there are no concerns of whether to report them.
\end{abstract}

Supplementary information The online version of this article (https:// doi.org/10.1038/s41431-019-0476-4) contains supplementary material, which is available to authorized users.

$\checkmark$ Ludwig Czibere

1.czibere@labor-becker.de

1 Laboratory Becker \& Colleagues, Führichstr. 70, 81671 Munich, Germany

2 TIB Molbiol GmbH, Eresburgstr. 22-23, 12103 Berlin, Germany

3 Department of Operative/Restorative Dentistry, Periodontology and Pedodontics, Ludwig Maximilian University Munich, Goethestr. 70, 80337 Munich, Germany

4 Pediatric Nephrology, RoMed Klinikum Rosenheim, Pettenkoferstr. 10, 83022 Rosenheim, Germany

5 Institute of Human Genetics, University of Cologne, Kerpener Str. 34, 50931 Cologne, Germany

6 Division of Pediatric Neurology, Center for Neuromuscular Disorders in Childhood. Dr. von Hauner Children's Hospital, Ludwig-Maximilians-Universität München, Lindwurmstr. 4, 80337 Munich, Germany

\section{Introduction}

Newborn screening (NBS) is a well-established public health service in many countries [1]. NBS enables early detection and treatment of a wide variety of diseases by analyzing metabolites, hormones, and enzyme activities, using dried blood spots (DBS). Sampling of the DBS occurs 1-2 days after birth. In NBS, diseases should only be screened for, if specific treatment is available [2-4]. Based on the often-cited Wilson and Jungner criteria [4], another principle of NBS is not to report unaffected carriers.

Spinal muscular atrophy (SMA; OMIM \#: 253300) is a severe, autosomal-recessive neuromuscular disease, characterized by the degeneration of $\alpha$-motor neurons $[5,6]$. The Caucasian population shows an incidence of 1:6000-1:10,000 [5-7], with estimates for Germany up to 1:4000 [8]. Based on direct genetic testing of SMN1 copies in a control population in Germany, a carrier frequency of 1:35 was found, resulting in an incidence of 1:4900 [9].

SMA is primarily caused by variants in the survival motor neuron $(S M N)$ gene. The gene consists of nine exons and 


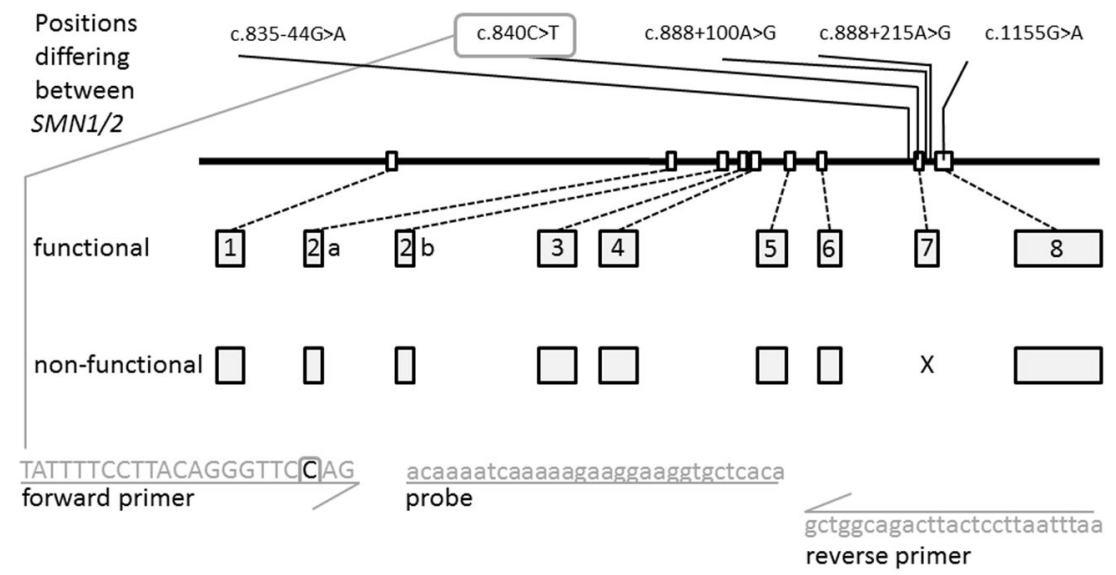

Fig. 1 Schematic overview of the $S M N$-coding genes. $S M N 1$ is encoded on chromosome 5 by nine exons (exons 2 and 3 are usually referred to as exon $2 \mathbf{a}$ and $2 \mathbf{b}$, respectively [12]). Exon 7 (the eighth exon) is missing in most SMA patients, and is missing from SMN2 transcripts as well. $S M N 1$ and $S M N 2$ only differ by five nucleotides as indicated [c.835-44G >A (rs1454173648); c.840C $>\mathrm{T}$ (rs1164325688);

encodes a $38-\mathrm{kD}$ SMN protein, which plays a key role in the assembly and regeneration of small nuclear ribonuclear proteins, mRNA splicing, and axonal RNA transport. The $S M N$ gene is located on chromosome 5 containing two highly homologous copies of $S M N$, a telomeric $(S M N 1)$ and a centromeric copy (SMN2) that differ by five bases altogether (Fig. 1) [10-12]. The transcription of SMN1 results in fulllength mRNA. In contrast, SMN2 transcription generates a shortened mRNA lacking exon 7 (c.840C $>\mathrm{T}$, a paralog sequence variant; Supplementary Table 1) with a small amount of full-length transcript also generated (Fig. 1).

Ninety-five percent of SMA-affected individuals have a homozygous deletion involving SMN1 exons 7 and 8, or exon 7 only. The latter is true for around 5\%, and represent SMN1/SMN2 hybrid genes [10-13]. Further, diseasecausing variants, like subtle variants $(3.4 \%)$ and no de novo variants (2\%) in the SMN1 gene, contribute to the incidence $[14,15]$. The $S M N 2$ gene is unable to compensate for the homozygous loss of $S M N 1$, but $S M N 2$ copy number is a well-established modifier on the variable SMA phenotype $[5,6,9,10]$.

SMA is classified into different phenotypes, based on the age of onset and the motor function achieved. SMA type I is the most severe and common type, which accounts for about $50 \%$ of patients. Life expectancy is generally considered to be $<2$ years, due to the rapid progression of muscle weakness and respiratory failure $[5,6,16]$. Although a therapy has recently become available $[5,6,17-20]$, the success of this therapy strongly depends on early intervention, because substantial loss of motor neurons occurs by 3 months of age and progresses during the course of the disease [16, 21]. Only a genetic test allows identification before the onset of symptoms and offers a treatment in time. c. $888+100 \mathrm{~A}>\mathrm{G} \quad(\mathrm{rs} 212214) ; \quad$ c. $888+215 \mathrm{~A}>\mathrm{G} \quad(\mathrm{rs} 1244569826) ;$ c.1155G $>$ A (rs1208416968)]. All variants shown are described using the NM_000344.3 transcript reference sequence based on NG_008691.1 for intron positions. Primers and probes for the c.840 site are indicated in gray; the site itself is indicated by a gray square in the forward primer

Challenges presented by NBS genetic testing include the extraction of sufficient DNA and selection of an appropriate assay to detect the $S M N 1$ deletion. The pitfall in selecting an appropriate assay for SMN1 deletions is the high homology to $S M N 2$. In addition, homologous recombination between the two genes can occur and might result in false-positive results, if positions beyond exon 7 are screened (Fig. 1) [22]. Further, a cleanup procedure for DNA from DBS had to be established and validated, addressing the questions of contamination, and intra- and interassay variability. We aimed to establish a protocol suitable to (i) extract DNA from standard DBS in a rapid way that can be used for testing in multiplex quantitative (q)PCR assays and further downstream applications, (ii) screen up to 2000 samples a day with one person operating the molecular genetic screening procedure and the least possible time occupying quantitative polymerase chain reaction (qPCR) cyclers, (iii) identify and eliminate possible sources and effects of contamination, and (iv) avoid falsepositive results by optimal assay design.

In addition, we demonstrate the functionality of the protocol on over 210,000 DBS extracted and analyzed, simultaneously generating the first data for SMA in a NBS setting for a largely Caucasian population.

\section{Materials and methods}

\section{Samples and preparation of DBS}

The SMA screening has been approved by the local ethics commission (Ethics Commission No. 16125 of the Bayerische Landesärztekammer). Throughout the screening procedure, samples were mainly recruited from hospitals, with 
about $42 \%$ of the samples originating from the State of North Rhine Westphalia (Northwest Germany) and 58\% from Bavaria (South Germany). Parents were informed and required to decide if they wanted to participate in the study. We acquired 3.2-mm punches from DBS cards (TFN, Ahlstrom-Munksjö, Bärenstein, Germany) using the Panthera Puncher 9 (Perkin Elmer, Waltham, MA, USA). To rule out the risk of a plate mixup, negative controls were used alternating between various positions on each plate. Special adaptors were required to fit 96-well semi-skirted PCR plates (4ti-0770/C, 4titude, Surrey, UK). Plates were sealed using a qPCR seal (4ti-0560, 4titude) for transport. For additional experiments, DBS was prepared and air dried for $48 \mathrm{~h}$ from fresh blood samples from anonymous donors.

\section{Washing of DBS punches and DNA elution (CXCE- buffer cleanup)}

All pipetting steps were performed using the ViaFlo96 system (Integra Biosciences, Zizers, Switzerland). DBS punches were incubated in 96-well plates in $50 \mu \mathrm{l}$ of water for $10 \mathrm{~min}$ on a plate shaker at $200 \mathrm{rpm}$ (IKA Labortechnik, Staufen, Germany); then $150 \mu \mathrm{l}$ of CX buffer ( $1 \times$ PBS and $0.5 \%$ Thesit ${ }^{\oplus}$ ) was added and incubated for a further $10 \mathrm{~min}$ on the plate shaker at $\sim 200 \mathrm{rpm}$. After a centrifugation step (Rotanta 460, Hettich $\mathrm{GmbH}$, Tuttlingen, Germany) at $1000 \mathrm{rpm}$ for $5 \mathrm{~min}$, the supernatant was removed with a vacuum device (VACUSAFE, Integra), and further, $150 \mu \mathrm{l}$ of $\mathrm{CX}$ buffer was added. Following another centrifugation step as described above, the supernatant was removed, and $150 \mu \mathrm{l}$ of water was added to remove residual PBS. After centrifugation and removal of the water, $50 \mu$ of CE buffer (10 mM Tris, $0.25 \mathrm{mM}$ EDTA, and $2 \mathrm{mM} \mathrm{NaOH}$ ) was added to each well, and the plates were sealed using a PCR seal (4ti-0500, 4titude). The plates were then incubated in a thermal cycler (Applied Biosystems 2720, Foster City, CA) for $10 \mathrm{~min}$ at $92{ }^{\circ} \mathrm{C}$.

\section{Primers and probes}

An 82-bp fragment of SMN1 exon 7 was amplified, as described by Strom et al. [23], using a slightly modified forward primer specifically targeting the SMN1 c.840C position (5'-TAT TTT CCT TAC AGG GTT CCA G-3'). A reverse primer (5'-GCT GGC AGA CTT ACT CCT TAA TTT AA- $\left.3^{\prime}\right)$ and a R6G-labeled probe (5'-F-ACC AAA TCA AAA AGA AGG AAG GTG CTC ACA-Q-3') bind to SMN1 and to SMN2. Since SMA detection is based on failure of the SMN1 exon 7 amplification, co-amplification of a reference gene is required to control the PCR reaction. The test can be combined with any genomic assay like betaactin (TIB Molbiol 66-0913-96). For this study, a TaqMan assay for cystinosin (CTNS, OMIM\# 606272) was used as control (Fleige et al., submitted) in a multiplexed qPCR. qPCR was performed in $10-\mu l$ reactions with LightCycler DNA Multiplex Master (Roche, Mannheim, Germany) in 384-well plates (Roche).

\section{qPCR setup}

qPCR reactions were set up by dispensing aliquoted primer/ probe mixes and enzymes from semi-skirted PCR plates to 384-well qPCR plates using the ViaFlo96 system. Samples from the 96-well DNA plates were dispensed to the 384-well plates in a predefined manner. qPCR was performed using a 384-well block on a LightCycler 480II (Roche), with initial denaturation at $95^{\circ} \mathrm{C}$ for $10 \mathrm{~min}$, followed by 45 cycles in three steps at $95{ }^{\circ} \mathrm{C}$ for $5 \mathrm{~s}, 60^{\circ} \mathrm{C}$ for $10 \mathrm{~s}$, and $72^{\circ} \mathrm{C}$ for $15 \mathrm{~s}$. A single acquisition of fluorescence signals was included in the $60{ }^{\circ} \mathrm{C}$ step. Sample lists were generated from the punching log files, converted to fit the 384-well plates, and imported directly to the LightCycler.

\section{qPCR evaluation}

qPCR experiments were evaluated based on cycle quantification $(\mathrm{Cq})$ applying the second derivate maximum analysis of the LightCycler software. An SMN1 gene copy was considered present, if a product was detected before cycle 36 (Cq-value $<36$ ), while the internal control was considered present if a Cq-value $<34$ was detected. If no product was visible in any assay, the result was invalid. If only the control assay showed sufficient product, the result was considered positive with the patient likely to have a homozygous deletion of SMNI exon 7. In both cases, fresh DBS punches were retested (internal verification). In case of a verified positive result for SMA in our laboratory, parents were asked to consult a specialized treatment center (Center for Neuromuscular Disorders in Childhood at the Dr von Hauner Children's Hospital in Munich, Neuropediatric Department of the University Hospitals of Essen, or Münster), where a blood sample was taken and sent to an independent laboratory for external confirmation of the screening result. In addition, an MLPA analysis was performed to determine $S M N 2$ copy numbers [24].

\section{Comparison of the CXCE-buffer cleanup with MagnaPure96 extraction}

Three punches were homogenized in $600 \mu \mathrm{l}$ of PBS using 1.4-mm zirconium beads (Precellys, Bertin Technologies, Montigny le Bretonneux, France) in a Precellys 24 homogenizer. Two-hundred microliters of the homogenized punches (corresponding to one punch) were extracted and eluted in $50 \mu \mathrm{l}$ of elution buffer on a MagnaPure96 system (Roche), using the MagnaPure small-volume nucleic acid 
extraction kit. One punch was subjected to the CXCE-buffer cleanup. Extractions were performed in eight replicates.

DNA quality and quantity were assessed based on the sample's performance in the SMA assay on the LightCycler and further routine assays (supplementary material).

\section{Robustness}

Robustness of the DNA elution method was addressed by retesting samples that had been tested invalid for the internal control $(\mathrm{Cq}>34)$ in the first qPCR during our routine screening. We collected 35 of such DNA samples from 4 consecutive weeks and repeated the qPCR.

\section{Evaluation of cross-contamination and precision}

We aimed to identify possible contamination sources, i.e., the punching process or the reusing of pipette tips during the different washing steps. The punching procedure was analyzed by punching from empty cards and cards prepared with blood in an alternating manner. A 96-well PCR plate was filled with punches according to the following pattern: column A-empty; column B-blood; column C-empty, etc. For one plate, the automated (plate A) Panthera Puncher 9 was used; the other plate was punched manually (plate $\mathrm{M}$ ), avoiding all possible contamination by punching the empty cards first and then the cards containing blood. For DNA preparation, fresh pipette tips were used for every step for plate A, including removal of the supernatant. According to the routine procedure, pipette tips were not changed during the washing steps for plate $\mathrm{M}$. Extracts were analyzed using the SMA test.

All DBS punches were prepared from the identical DBS; thus, the values are also representative for intra- and interassay variability.

\section{Statistics}

Cq-values are presented as means \pm standard error of means (SEM). The statistical significance $(p<0.05)$ of the differences between the experimental groups was tested using the $t$ test, corrected according to Bonferroni-Holm [25]. Confidence interval (CI) calculations were performed using MedCalc for Windows, version 19.0 (MedCalc Software, Ostend, Belgium).

\section{Results}

\section{Comparison of the CXCE-buffer cleanup method with MagnaPure96 extraction}

MagnaPure96 extraction resulted in a slightly but not statistically significant increased amount of DNA. On average,
CXCE-buffer cleanup resulted in Cq-values of $31.85 \pm 0.10$, which was approximately one Cq higher compared with the extraction performed on a MagnaPure96 (30.90 \pm 0.07 ; Fig. 2).

\section{Robustness}

Altogether, no sample has been reported invalid so far, as in all cases of invalid results, processing of a fresh DBS delivered valid results. However, to elucidate the reason of invalid results in the first assessment, 35 samples have been collected over 4 weeks, showing an invalid result. qPCR was repeated using the original DNA. Out of these 35 DNA samples, only one delivered an invalid result. This suggests that incorrect pipetting in the PCR setup or incomplete sealing of the qPCR plate led to invalid results rather than a failure of the cleanup procedure.

\section{Evaluation of cross-contamination and precision}

All DBS punches were detected with Cq-values ranging from 30.73 to 32.96 on both plates. Plate A (automated punching) and Plate $M$ (manual punching) had several wells with empty punches that displayed a late $\mathrm{Cq}(\mathrm{Cq}>$ 37 ) in the SMA test. Plate A showed late Cqs in more negative wells than Plate M ( $46 \%$ vs. $13 \%$, respectively; Fig. 3). This indicates that automated punching is the main source of contamination. Variability shows a $\mathrm{Cq}$ of $31.36 \pm 0.09$ for 40 intra-assay technical replicates and $31.84 \pm 0.05$ for another 40 intra-assay technical replicates, reflecting an average variability below $1 \mathrm{Cq}$ for 40 replicates each. Thus, reproducibility is given for all samples $(N=80$; sensitivity $=1,95 \% \mathrm{CI}=0.91$; specificity $=1,95 \% \mathrm{CI}=0.91)$.

\section{Newborn screening}

To establish the SMN1 assay, blood samples from known SMA patients $(N=13)$ and a subset of their parents (heterozygous carriers; $N=8$ ) were analyzed. In addition, the samples were distributed on DBS cards and tested thereafter. All patients were identified as carrying the homozygous deletion, while the parents showed $\mathrm{Cq}<36$ for $S M N 1$ in the qPCR test $(N=21$; sensitivity $=1,95 \% \mathrm{CI}=$ 0.75 ; specificity $=1,95 \% \mathrm{CI}=0.63$ ).

The NBS for SMA started mid-January 2018. So far, 213,279 DBS have been analyzed for a homozygous deletion of exon 7 of SMN1. Thirty samples showed amplification of the reference gene, but no amplification of $S M N 1$; thus a homozygous deletion of exon $7(N=$ 213,279; sensitivity $=1,95 \% \mathrm{CI}=0.88$; specificity $=1$, $95 \% \mathrm{CI}=1)$. All 30 cases identified were referred to a neuropediatric center for consultation and were retested for 


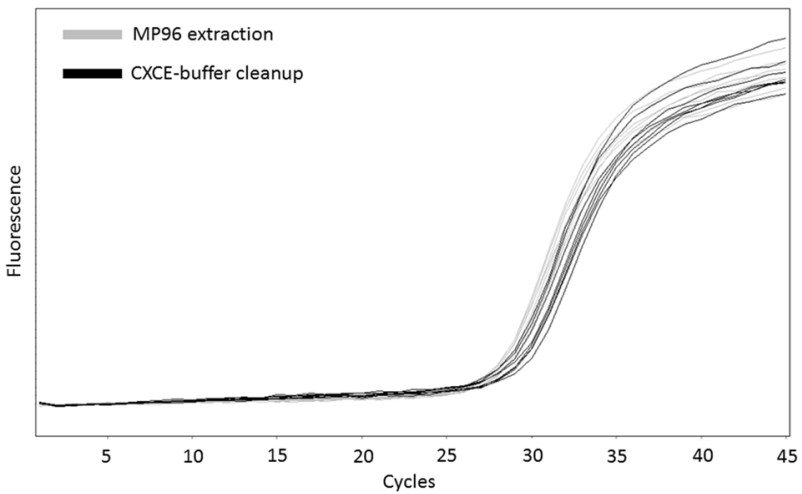

Fig. 2 Performance comparison of the CXCE-buffer cleanup vs. extraction of nucleic acids on a MagnaPure96. The amplification curves show the performance of nucleic acids of both extraction methods from the same sample (gray lines: MagnaPure96 extraction, black lines: CXCE-buffer cleanup) in the SMN1 exon 7 deletion qPCR

\section{Plate A}

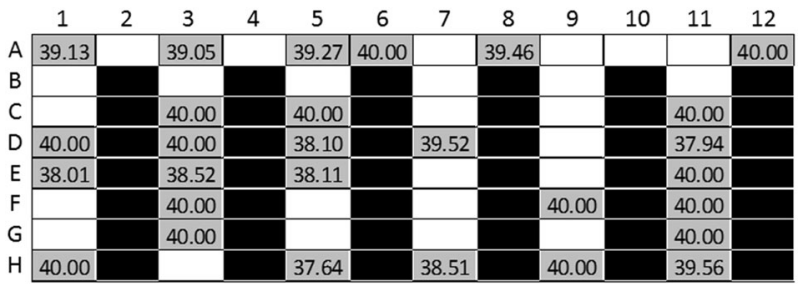

Plate M

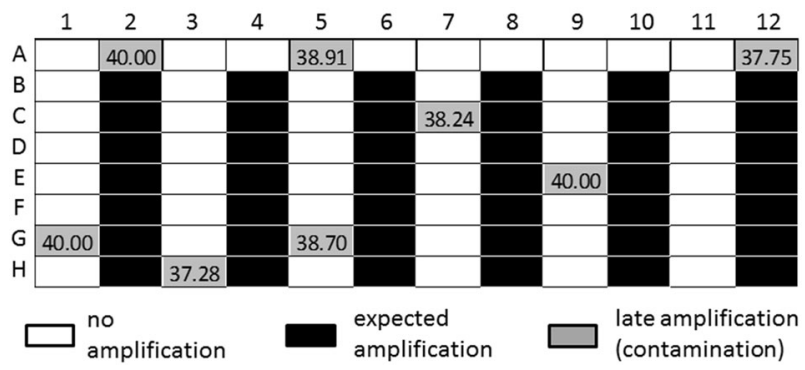

Fig. 3 Schematic contamination patterns on a 96-well plate. Plate A shows the results of automated punching, Plate $M$ from manual punching with white wells: no amplification, black wells: expected amplification, and gray wells: late amplification, i.e., contamination including the respective Cq-values

the exon 7 deletion and their copy numbers of SMN2. Our primary screening results were confirmed in all cases by MLPA analysis from fresh blood samples in an independent laboratory (Genetikum, Neu-Ulm, Germany). The copy numbers for $S M N 2$ varied between 2 and 4 (Vill et al., submitted). To date, no false-positive results have been observed; no false-negative results have been reported to us either. To date, we have determined the status of all samples from the original DBS, without any sample reported invalid.

\section{Discussion}

SMA is the second most common autosomal recessively inherited disease in humans after cystic fibrosis [26]. To include this life-threatening condition in NBS, we developed an efficient genetic screening procedure.

We established a rapid and inexpensive method implemented in a workflow that allowed us to screen a high number of samples in the shortest possible turnaround time (within $24 \mathrm{~h}$ per sample).

Our CXCE-buffer cleanup procedure resulted in eluates containing enough DNA for retesting, or further genetic assays like severe combined immunodeficiency (SCID), with no special reagents or commercial kits required. We limited the risk of sample mixups, a common pitfall in laboratory medicine [27], by keeping the DBS punches in the same well of a 96-well plate from arrival of the samples to the setup of the qPCR assays.

Our study showed storage conditions of NBS cards did not affect the quality of results. Automated punching of DBS is commonly used in NBS. We demonstrated that trace amounts of DNA are transferred into neighboring wells of a 96-well plate, and that this could not be avoided without any additional laborious and time-consuming steps. Thus, the typical range for $\mathrm{Cq}$-values had to be established in the first qPCR assays and confirmed during validation to determine the appropriate cutoffs for late Cq-values. This was straightforward for the SMN1 assay, since a Cq difference $\geq 3$ was detected between contaminations and heterozygous or homozygous wild-type samples.

Our results show the importance of considering the contamination problem of automated punching for genetic screening procedures.

The CXCE-buffer cleanup delivered reliable results from qPCR reactions and for further molecular genetic approaches. The number of samples requiring repeated testing due to technical errors was very low $(<1 \%)$. Repeated cleanup and qPCR from fresh DBS resolved the error in all cases; therefore, no invalid result had to be reported. In addition, the data for intra- and interassay variability showed stable reproducibility of the results. Using the MagnaPure96, an automated, costly, and time-consuming extraction system, the DNA yield was only slightly higher. The CXCE-buffer cleanup is an easy DBS cleanup procedure that can be used for various assays. Combining the cleanup with a 384-well qPCR setup, limited the required qPCR capacity to one cycler; thus, over 1000 reactions could be processed in three runs in $<5 \mathrm{~h}$.

Among others, Saavedra-Matiz et al. described a similar method for DNA cleanup [28]. The same protocol was applied for a pilot- screening study of SMA in the New York state [29]. Many other well-designed studies dealt with DNA extraction for NBS, but have not described the 
screening of a large population subset or a complete setup for routine screening from punching to $\mathrm{qPCR}$ [13, 28, 30, 31].

Prior et al. described two pilot studies for SMA NBS, giving detailed instructions for sample handling, but limited to a commercial kit for DNA cleanup and to a 96-well format [13]. Taken together, none of the previous studies described a procedure that can be reproduced in a 384-well format.

Few other large-scale genetic NBS projects have been published so far; among others, a study of genetic screening for hearing loss assessing three simple nucleotide variants and a single-base deletion in over 140,000 samples. Genetic screening for hearing loss was implemented to increase the sensitivity of the physical test for hearing loss [32].

A study from Taiwan described the screening of 120,267 newborns for SMA. Within 22 months, 15 potential cases were identified, where eight turned out to be false positive. The introduction of a second-tier digital droplet (dd)PCR assay was necessary to accurately diagnose SMA from DBS without false positives. In the Taiwanese study, the SMNIspecific c. $888+100 \mathrm{~A}$ site was targeted for screening. However, in two cases, the targeted site was homozygous c. $888+100 \mathrm{~A}>\mathrm{C}$; therefore, no product was visible. In five further cases, the $S M N 1$ gene sequence was converted to $S M N 2$ after the c.840C site [22]. Targeting the c.840C site eliminates these possibilities of receiving false-positive results and avoids second-tier testing [23, 33] for NBS, while still detecting only homozygous deletions, i.e., affected newborns.

With our protocol, we were able to screen 213,279 samples from routine NBS within 18 months. Thus, we provide the first unbiased population-based screening for SMNI exon 7 deletions for a Caucasian population. Based on the described incidence of the disease between 1:4000 and 1:10,000, 20-50 cases were expected for 200,000 samples. In fact, we found 30 cases with all cases confirmed by an independent laboratory from fresh blood samples (100\% positive predictive value). Unknown or de novo variants in the primer and probe sequences remain as risk for false-positive results. No missed patients have been reported to us to date (sensitivity: 100\%; Vill et al., submitted). However, we expect to encounter falsenegative results in the future, as other pathogenic variants are not detected with this assay design. This would affect $\sim 3-5 \%$ of SMA cases [12].

MLPA analysis or digital droplet PCR for determination of SMN2 copy number seems to be feasible from newborn blood spots in a two-tier protocol. However, with this lethal condition, it is mandatory to confirm the first result from a fresh blood sample. This confirmatory testing including copy number determination was performed in the independent laboratory. SMN2 copy numbers varied between 2 and 4 for the identified patients. Disease severity has been shown to correlate with SMN protein levels, and SMA Type 0, I, II, or III has shown a significant correlation to $S M N 2$ copy number. In one study, $96 \%$ of individuals with Type I carried one or two SMN2 copies, whereas $100 \%$ of individuals with Type III carried three or four copies [9]. However, the SMA degree type does not show a complete correlation with the copy numbers of $S M N 2$ being present [16], as variants other than the aforementioned deletion of exon 7 may contribute to different phenotypic outcomes. Discordances have been reported in the patients with 3-4 copies of $S M N 2$, who may develop SMA Type I, II, or III or rarely remaining even asymptomatic [9, 34, 35]. Recently, a therapy (Nusinersen $\left(\right.$ Spinraza $\left.^{\mathrm{TM}}\right)$ ) for SMA has been approved by health authorities (U.S. Food and Drug Administration (FDA): December 2016, European Medicines Agency: May 2017). Notably, Nusinersen is among the first four therapeutics licensed for clinical use, based on antisense oligonucleotides [36]. Application results in more of the SMN2 fulllength transcript, which compensates for the loss of SMN1. All identified patients from this study were consulted by clinicians, with treatment advised to all patients, with two or three copies of SMN2 [37].

Other therapeutics are still in clinical phase 1 or 2 trials, with another therapy (AVXS-101) aiming to replace SMN1 [38]. SMA has been added to the Recommended Universal Screening Panel in the United States for NBS as of July 2018. Genetic screening is already offered in some countries/states for SCID screening. From a purely technical point of view, it is not a classical genetic screening, as no DNA sequence data are revealed. SCID is a disorder of (T-cell) lymphocyte formation, where in affected patients, no formation of the so-called T-cell receptor excision circles takes place. However, these DNA circles are found in healthy individuals, where they can be detected. Combined assays for SMA and SCID have already been tested in previous studies [30]. With the screening for SMA and SCID, we are at the dawn of a new era of primary DNA-based NBS. However, to screen for multiple loci will require further development in the field.

Acknowledgements We thank Peta Snikeris for proofing the paper.

Funding The screening was funded by the German Cystinosis Foundation (Cystinose Stiftung, DSZ-Regional Office Munich, Widenmayerstr. 10, 80538 Munich, Germany). The funders had no influence on the design, interpretation, and publication of these data.

\section{Compliance with ethical standards}

Conflict of interest The authors declare that they have no conflict of interest. 
Publisher's note: Springer Nature remains neutral with regard to jurisdictional claims in published maps and institutional affiliations.

\section{References}

1. Therrell BL, Padilla CD, Loeber JG, Kneisser I, Saadallah A, Borrajo GJ, et al. Current status of newborn screening worldwide: 2015. Semin Perinatol. 2015;39:171-87.

2. Andermann A, Blancquaert I, Beauchamp S, Costea I. Guiding policy decisions for genetic screening: developing a systematic and transparent approach. Public Health Genom. 2011;14:9-16.

3. Andermann A, Blancquaert I, Beauchamp S, Dery V. Revisiting Wilson and Jungner in the genomic age: a review of screening criteria over the past 40 years. Bull World Health Organ 2008;86:317-9.

4. Dobrow MJ, Hagens V, Chafe R, Sullivan T, Rabeneck L. Consolidated principles for screening based on a systematic review and consensus process. CMAJ 2018;190:E422-9.

5. Finkel RS, Mercuri E, Meyer OH, Simonds AK, Schroth MK, Graham RJ, et al. Diagnosis and management of spinal muscular atrophy: part 2: pulmonary and acute care; medications, supplements and immunizations; other organ systems; and ethics. Neuromuscul Disord. 2018;28:197-207.

6. Mercuri E, Finkel RS, Muntoni F, Wirth B, Montes J, Main M, et al. Diagnosis and management of spinal muscular atrophy: Part 1: Recommendations for diagnosis, rehabilitation, orthopedic and nutritional care. Neuromuscul Disord. 2018;28:103-15.

7. Sugarman EA, Nagan N, Zhu H, Akmaev VR, Zhou Z, Rohlfs EM, et al. Pan-ethnic carrier screening and prenatal diagnosis for spinal muscular atrophy: clinical laboratory analysis of $>72,400$ specimens. Eur J Hum Genet. 2012;20:27-32.

8. Verhaart IEC, Robertson A, Leary R, McMacken G, Konig K, Kirschner J, et al. A multi-source approach to determine SMA incidence and research ready population. $J$ Neurol. 2017;264:1465-73.

9. Feldkötter M, Schwarzer V, Wirth R, Wienker TF, Wirth B. Quantitative analyses of SMN1 and SMN2 based on real-time LightCycler PCR: fast and highly reliable carrier testing and prediction of severity of spinal muscular atrophy. Am J Hum Genet. 2002;70:358-68.

10. Pyatt RE, Prior TW. A feasibility study for the newborn screening of spinal muscular atrophy. Genet Med. 2006;8:428-37.

11. Lefebvre S, Burglen L, Reboullet S, Clermont O, Burlet P, Viollet $\mathrm{L}$, et al. Identification and characterization of a spinal muscular atrophy-determining gene. Cell. 1995;80:155-65.

12. Wirth B. An update of the mutation spectrum of the survival motor neuron gene (SMN1) in autosomal recessive spinal muscular atrophy (SMA). Hum Mutat. 2000;15:228-37.

13. Prior TW, Snyder PJ, Rink BD, Pearl DK, Pyatt RE, Mihal DC, et al. Newborn and carrier screening for spinal muscular atrophy. Am J Med Genet A. 2010;152A:1608-16.

14. Wirth B, Schmidt T, Hahnen E, Rudnik-Schoneborn S, Krawczak M, Muller-Myhsok B, et al. De novo rearrangements found in 2\% of index patients with spinal muscular atrophy: mutational mechanisms, parental origin, mutation rate, and implications for genetic counseling. Am J Hum Genet. 1997;61:1102-11.

15. Wirth B, Herz M, Wetter A, Moskau S, Hahnen E, RudnikSchoneborn S, et al. Quantitative analysis of survival motor neuron copies: identification of subtle SMN1 mutations in patients with spinal muscular atrophy, genotype-phenotype correlation, and implications for genetic counseling. Am J Hum Genet. 1999;64:1340-56.

16. Kolb SJ, Kissel JT. Spinal muscular atrophy. Neurol Clin. 2015;33:831-46.
17. Finkel RS, Chiriboga CA, Vajsar J, Day JW, Montes J, De Vivo DC, et al. Treatment of infantile-onset spinal muscular atrophy with nusinersen: a phase 2, open-label, dose-escalation study. Lancet. 2016;388:3017-26.

18. Messina S. New directions for SMA therapy. J Clin Med. 2018;7:251

19. Alcantara-Ortigoza MA, Belmont-Martinez L, Vela-Amieva M, Gonzalez-Del Angel A. Analysis of the CTNS gene in nephropathic cystinosis Mexican patients: report of four novel mutations and identification of a false positive 57-kb deletion genotype with LDM-2/exon 4 multiplex PCR assay. Genet Test. 2008;12:409-14.

20. Mercuri E, Darras BT, Chiriboga CA, Day JW, Campbell C, Connolly AM, et al. Nusinersen versus Sham Control in Later-Onset Spinal Muscular Atrophy. N Engl J Med. 2018;378:625-35.

21. Saffari A, Kolker S, Hoffmann GF, Weiler M, Ziegler A. Novel challenges in spinal muscular atrophy - How to screen and whom to treat? Ann Clin Transl Neurol. 2019;6:197-205.

22. Chien YH, Chiang SC, Weng WC, Lee NC, Lin CJ, Hsieh WS, et al. Presymptomatic diagnosis of spinal muscular atrophy through newborn screening. J Pediatr. 2017;190:124-9.

23. Strom CM, Anderson B, Peng M, Patel U, Braastad CD, Sun W. 1000 sample comparison of MLPA and RT-PCR for carrier detection and diagnostic testing for spinal muscular atrophy type 1. Open J Genet. 2013;3:111-4.

24. Arkblad EL, Darin N, Berg K, Kimber E, Brandberg G, Lindberg $\mathrm{C}$, et al. Multiplex ligation-dependent probe amplification improves diagnostics in spinal muscular atrophy. Neuromuscul Disord. 2006;16:830-8.

25. Forst HT. Problems of multiple tests and evaluations in drug research. Arzneim Forsch. 1985;35:563-9.

26. Phan HC, Taylor JL, Hannon H, Howell R. Newborn screening for spinal muscular atrophy: anticipating an imminent need. Semin Perinatol. 2015;39:217-29.

27. Durner J. Clinical chemistry: challenges for analytical chemistry and the nanosciences from medicine. Angew Chem Int Ed Engl. 2010;49:1026-51

28. Saavedra-Matiz CA, Isabelle JT, Biski CK, Duva SJ, Sweeney ML, Parker AL, et al. Cost-effective and scalable DNA extraction method from dried blood spots. Clin Chem. 2013;59:1045-51.

29. Kraszewski JN, Kay DM, Stevens CF, Koval C, Haser B, Ortiz V, et al. Pilot study of population-based newborn screening for spinal muscular atrophy in New York state. Genet Med. 2018;20:608-13.

30. Taylor JL, Lee FK, Yazdanpanah GK, Staropoli JF, Liu M, Carulli JP, et al. Newborn blood spot screening test using multiplexed real-time PCR to simultaneously screen for spinal muscular atrophy and severe combined immunodeficiency. Clin Chem. 2015;61:412-9.

31. Ar Rochmah M, Awano H, Awaya T, Harahap NIF, Morisada N, Bouike Y, et al. Genetic screening of spinal muscular atrophy using a real-time modified COP-PCR technique with dried bloodspot DNA. Brain Dev. 2017;39:851-60.

32. Hao Z, Fu D, Ming Y, Yang J, Huang Q, Lin W, et al. Large scale newborn deafness genetic screening of 142,417 neonates in Wuhan, China. PLoS ONE. 2018;13:e0195740.

33. Smith M, Calabro V, Chong B, Gardiner N, Cowie S, du Sart D. Population screening and cascade testing for carriers of SMA. Eur J Hum Genet. 2007;15:759-66.

34. Calucho M, Bernal S, Alias L, March F, Vencesla A, RodriguezAlvarez FJ, et al. Correlation between SMA type and SMN2 copy number revisited: an analysis of 625 unrelated Spanish patients and a compilation of 2834 reported cases. Neuromuscul Disord. 2018;28:208-15. 
35. Oprea GE, Krober S, McWhorter ML, Rossoll W, Muller S, Krawczak M, et al. Plastin 3 is a protective modifier of autosomal recessive spinal muscular atrophy. Science. 2008;320:524-7.

36. Crooke ST, Witztum JL, Bennett CF, Baker BF. RNA-targeted therapeutics. Cell Metab. 2018;27:714-39.

37. Glascock J, Sampson J, Haidet-Phillips A, Connolly A, Darras B, Day J, et al. Treatment algorithm for infants diagnosed with spinal muscular atrophy through newborn screening. J Neuromuscul Dis. 2018;5:145-58.

38. Mendell JR, Al-Zaidy S, Shell R, Arnold WD, RodinoKlapac LR, Prior TW, et al. Single-dose gene-replacement therapy for spinal muscular atrophy. N Engl J Med. 2017; 377:1713-22. 